Relations industrielles

Industrial Relations

\title{
Economic Consultative Bodies : Their Origins and Institutional Characteristics, by Paul Malles, édité par Information Canada, Ottawa, Economic Council of Canada, 1971, 239 pp.
}

\section{André Roy}

Volume 26, numéro 4, 1971

URI : https://id.erudit.org/iderudit/028281ar

DOI : https://doi.org/10.7202/028281ar

Aller au sommaire du numéro

Éditeur(s)

Département des relations industrielles de l'Université Laval

ISSN

0034-379X (imprimé)

1703-8138 (numérique)

Découvrir la revue

Citer ce compte rendu

Roy, A. (1971). Compte rendu de [Economic Consultative Bodies : Their Origins and Institutional Characteristics, by Paul Malles, édité par Information Canada, Ottawa, Economic Council of Canada, 1971, 239 pp.] Relations industrielles / Industrial Relations, 26(4), 1059-1060. https://doi.org/10.7202/028281ar

Tous droits réservés @ C Département des relations industrielles de l'Université Laval, 1971
Ce document est protégé par la loi sur le droit d'auteur. L'utilisation des services d'Érudit (y compris la reproduction) est assujettie à sa politique d'utilisation que vous pouvez consulter en ligne.

https://apropos.erudit.org/fr/usagers/politique-dutilisation/ 
liams. En plus des raisons ci-haut mentionnées à cet effet, nous ajoutons que le texte se lit très bien, ceci étant dû principalement à la clarté et à la qualité du style.

\section{Bertrand BELZILE}

The Best of the Canadian Personnel Journal, by J.R. Perigoe and T.F. Hercus, Toronto, Canadian Personnel and Industrial Relations Journal, 1971, $325 \mathrm{pp}$.

Le Canadian Personnel and Industrial Relations Journal a réussi dans cet ouvrage les meilleurs articles que cette revue mensuelle a publiés depuis seize ans. Ces cinquante articles sont répartis sous la classification suivante : gestion du personnel ; organisation ; sélection ; formation; salaires ; hygiène industrielle; relations du travail ; communications internes; professionnalisation ; éducation permanente. On a aussi ajouté quelques articles en langue française.

La plupart des articles ont pour auteur des praticiens de la gestion du personnel. L'ensemble de ces points de vue permet de découvrir les préoccupations, l'orientation ainsi que l'évolution du climat qui règne au Canada dans ce domaine important des relations du travail.

\section{Gérard DION}

\section{Economic Consultative Bodies : Their} Origins and Institutional Characteristics, by Paul Malles, édité par Information Canada, Ottawa, Economic Council of Canada, 1971, 239 pp.

Les corps économiques consultatifs se sont développées énormément au cours des dernières années, principalement depuis la fin de la deuxième guerre mondiale. On en retrouve aujourd'hui dans la plupart des pays industrialisés d'Occident.

Paul Malles, du Conseil économique du Canada, a voulu les faire connaître en répondant à certaines questions que se pose le public au sujet de ces organismes. Pourquoi ont-ils fleuri dans les pays industriels? Quels sont les motifs qui ont présidé à leur établissement? A quels besoins répondent-ils ?
L'auteur, s'inspirant d'études sur le sujet faites en Europe attribue leur généralisation au rôle de plus en plus grand que joue l'Etat dans la vie économique, d'où l'importance pour les gouvernements modernes d'obtenir la participation des différents groupes d'intérêts à l'élaboration de leurs politiques économiques en ce qui concerne le plein emploi, la stabilité des prix, l'équilibre des paiements internationaux et la participation effective de toutes les classes de la société à l'élévation des niveaux de vie.

Evidemment, le rôle et la composition en varient beaucoup d'un pays à l'autre selon les contextes historiques. A certains endroits, les conseils sont tripartites : Etat, employeurs et salariés; ailleurs, ils sont formés de l'ensemble des corps intermédiaires existants ; enfin, il arrive que les gouvernements se contentent de faire appel à des comités d'experts, comme c'est le cas notamment aux Etats-Unis.

Les conseils diffèrent également quant à la nature de leurs suggestions: dans certains cas, l'institution joue un rôle essentiellement consultatif en ce sens qu'elle limite son action à donner les conseils qui lui sont demandés sur un sujet donné, le gouvernement restant absolument libre de les accepter ou de les rejeter, tandis que, dans d'autres cas, un véritable dialogue s'engage entre le gouvernement et les corps intermédiaires.

En général, les conseils économiques poursuivent les objets suivants : apporter aux services de l'Etat des connaissances et une expérience qu'ils ne possèdent pas ; établir un consensus général au moment des prises de décision; effectuer des recherches approfondies sur divers problèmes économiques et sociaux; élever le niveau de la discussion sur les questions économiques dans le public.

Malles étudie ensuite le fonctionnement du système dans un certain nombre de pays, notamment en France, aux Pays-Bas, en Belgique, en Grande-Bretagne, en Suède, en Allemagne occidentale et aux Etats-Unis.

Tous ceux qui ont pour responsabilité d'instituer à l'intérieur du pays les mécanismes nécessaires à la démocratisation de la vie économique et sociale trouveront profit à étudier dans cet ouvrage les moyens utilisés par différentes na- 
tions, selon le sens de leur évolution politique et des idéologies sociales dominantes, pour se doter d'organismes consultatifs valables.

André ROY

Le Code du travail : sa nature, sa portée, ses effets, par Fernand Morin. Québec, Ministère du travail et de la main-d'oeuvre, 1971, 61 pp.

Ce petit ouvrage n'a aucune prétention scientifique. Il a simplement pour but de mettre à la portée de tous ceux qui sont engagés dans les relations du travail les points essentiels du Code du travail en vigueur actuellement au Québec.

Après une introduction où l'auteur explique brièvement la nature du droit du travail et décrit les principales mesures législatives en ce domaine, l'ouvrage comprend deux chapitres. Le premier traite des «parties en présence dans les rapports collectifs du travail : deux parties bien distinctes; la détermination du syndicat majoritaire $\gg$. Le second chapitre est consacré à «l'élaboration par les parties des conditions de travail: la négociation collective; la convention collective ». Le tout est suivi d'annexes comprenant la liste des lois du travail distribuées selon leur objet, un tableau synoptique du mécanisme prévu au Code du travail, un questionnaire récapitulatif et une brève bibliographie.

Les lecteurs de Relations industrielles connaissent bien l'auteur qui est depuis longtempps un des collaborateurs réguliers. Grâce à sa compétence en droit du travail, à son expérience et à ses grandes qualités de pédagogue, il a réussi à présenter un texte où il a su allier clarté, concision et disposition pour en faire un outil extrêmement utile à tous. On pourra avantageusement s'en servir dans des sessions d'étude à l'intention des travailleurs.

Gérard DION

\section{Dictionnaire des partis communistes et des mouvements révolutionnaires, par François Fejtö, Paris, Casterman, 1971, $238 \mathrm{pp}$.}

Il s'agit non pas d'un dictionnaire mais d'un répertoire par pays des partis, mouvements, organisations considérés comme communistes ou qui se donnent pour telles. Ce répertoire est accompagné quand ils sont disponibles de renseignements sur la date de la fondation, les leaders, les effectifs, la presse et l'orientation idéologique pro-soviétique ou pro-chinoise de ces partis, mouvements et organisations.

Un tel répertoire constitue en soi un instrument de travail utile même en relations industrielles puisqu'il peut se prêter à une étude plus poussée de l'influence que ces divers organisrnes pourraient exercer sur les syndicats du moins lorsqu'il s'agit de pays où le parti communiste n'est pas au pouvoir.

La lecture de ce répertoire confirme l'ampleur du «schisme» qui divise ce monde communiste entre «pro-soviétique » et «pro-chinois».

L'essai d'interprétation de ce schisme contenu dans les premières pages du livre n'est pas non plus sans intérêt. Les divers facteurs de dislocation de l'unité du monde communiste qui ne fut jamais complète même du vivant de Staline, y sont succintement abordés; ce sont le débat idéologique sur l'interprétation du marxisme-léninisme, et la stratégie du mouvement communiste, la remontée spectaculaire des nationalismes, et l'affrontement de l'Union soviéticue et de la Chine pour le contrôle du mouvement communiste international. A cet essai d'interprétation qui n'est qu'esquissé, il manque selon nous une donnée principale soulignée notamment par Raymond Aron: A la politique des blocs de la guerre froide a succédé une politique plus souple, à l'est comme à l'ouest, dès que le développement de l'arme thermonucléaire et de son vecteur principal, la fusée, balistique intercontinental a en quelque sorte dévalorisé l'importance stratégique des états tampons.

\section{Bernard SOLASSE}

GAP initiation pratique, par Dominique Lamure, Paris, Collection L'informathèque, Entreprise moderne d'édition, $1970,155 \mathrm{pp}$.

L'Entreprise moderne d'édition lance une nouvelle collection, l'Informathèque, sous la direction de MM. J.L. Groboillot et J.M. Dethoor du Centre de Calcul de la Chambre de Commerce et d'Industrie de Paris. Cette collection a pour objectif de fournir aux étudiants, 